\title{
COMPARACIÓN DEL AISLAMIENTO BACTERIANO POR MEDIO DE HISOPADO Y LAVADO DE BAJO VOLUMEN, EN YEGUAS CRIOLLAS COLOMBIANAS CON ENDOMETRITIS
}

\author{
Comparison of the bacterial isolation through swabbing and low \\ volume washing in Colombian Creole mares with endometritis
}

\author{
Renso Sneider Gallego Rodríguez ${ }^{1^{*}}$ \\ E-mail: renso1287@gmail.com
}

Juan Diego Lujan Giraldo²

E-mail: $\quad$ diego-322@hotmail.com

${ }^{1}$ MVZ, Esp, MSc. Grupo de Investigación Veterinaria GISCA, Facultad de Medicina Veterinaria y Zootecnia, Institución Universitaria Visión de las Américas, Medellin, Colombia.

${ }^{2} M V(e)$. Práctica privada y académica en medicina veterinaria, Medellín, Colombia

Fecha recepción: 25 de enero de 2021 / Fecha Aprobación: 22 de marzo de 2021 / Fecha Publicación: 29 de junio 2021

\section{RESUMEN}

La endometritis bacteriana en las yeguas es una de las principales causas de subfertilidad, y genera efectos negativos en el desarrollo de la reproducción equina. A causa de manejos reproductivos inadecuados, el diagnóstico y la terapéutica generalmente fracasan, razón por la cual aumentan los casos de infertilidad en las hembras. El presente estudio se realizó con 15 yeguas criollas colombianas, las cuales presentaron signos de subfertilidad asociados a abortos, retención placentaria, repetición de celos, secreción vulvar, entre otros. Los ejemplares fueron diagnosticados por medio de examen clínico reproductivo y de cultivo microbiológico, mediante las técnicas de hisopado uterino y de lavado de bajo volumen. Los datos fueron analizados mediante estadística descriptiva. Del total de muestras analizadas, $E$. coli fue el agente con mayor frecuencia de aislamiento de manera individual y en infecciones mixtas. A su vez, de las 15 muestras evaluadas para el cultivo, mediante la técnica de hisopado, se encontró que $E$. coli se aisló en un 33,33\% (5/15), mientras que, con el lavado de bajo volumen, se obtuvo un aislamiento de 13,33\% (2/15). De las técnicas analizadas, el hisopado mostró una mayor eficacia en el aislamiento de E. coli, con respecto al lavado de bajo volumen.

Palabras claves.

Bacterias, diagnóstico, hisopado, lavado, endometritis, yeguas.

\section{ABSTRACT}

The bacterial endometritis in mares is one of the main causes of subfertility, generating negative factors in the development of equine reproduction. Generally, due to inadequate reproductive management, the diagnosis and therapy fail, increasing cases of infertility in females. The present study was carried out with 15 Colombian Creole mares which presented such signs of subfertility as abortions, placental retention, repetition of the heat and the vulvar discharge among others. The animals were diagnosed via

\section{Cómo citar:}

Gallego Rodríguez, R. S. y Lujan Giraldo, J.D. (2021). Comparación del aislamiento bacteriano por medio de hisopado y lavado de bajo volumen, en yeguas criollas colombianas con endometritis. Revista Facultad de Ciencias Agropecuarias -FAGROPEC. Universidad de la Amazonia. Vol. 13 (1), 36-43. https://doi.org/10.47847/fagropec.v13n1a4 
reproductive clinical testing and a microbiological culture, using the uterine swabbing and low volume lavage techniques. The data were analyzed through the descriptive statistics. Of the total amount of samples analyzed, E. coli was the agent with the highest frequency of individual isolation and in mixed infections. At the same time, according to the 15 samples evaluated for culture by the swab technique, the $E$. coli was isolated in $33.33 \%(5 / 15)$ of them. Meanwhile the low volume washing technique showed an isolation of $13.33 \%(2 / 15)$. Concerning the analyzed techniques, the swabbing showed greater efficiency in isolating $E$. coli than the low volume washing.

\section{Key words}

bacteria, diagnosis, swab, lavage, endometritis, mares

\section{INTRODUCCIÓN}

La endometritis en yeguas es una de las patologías más frecuentes en la práctica veterinaria, y una de las principales causas de subfertilidad (Ferris, 2016). Esta alteración puede ser de origen bacteriano, fúngico o inmunomediada (Siemieniuch, 2019). Las endometritis bacterianas son problemáticas frecuentemente encontradas en la reproducción equina, y pueden ser derivadas de malas prácticas y de diagnósticos inadecuados, en los cuales la antibioticoterapia fracasa, aumentando así la infertilidad (Causey RC, 2008).

Las bacterias encontradas de manera frecuente en el endometrio de las yeguas son Streptococo equi zooepidermicus, Escherichia coli y Pseudomonas aeruginosa (Overbeck W, 2011); a su vez, han sido descritas otras bacterias con presentación común en los aislamientos, como es el caso de Klebsiella pneumoniae, Staphylococcus coagulasa-negativa y Proteus spp. (Terttu K, 2016). Generalmente, los signos reproductivos aparecen en yeguas que no quedan gestantes post servicio y que presentan reabsorción embrionaria, pérdidas fetales tempranas, abortos, placentitis o metritis postparto (LeBlanc, 2009).

El diagnóstico de la endometritis bacteriana se basa en el historial reproductivo del animal, sumado a la utilización de técnicas como el aislamiento del agente patógeno por medio del cultivo (Scoggin, 2016). La técnica de hisopado permite la toma de muestras desde varios puntos diferentes, en la superficie uterina; a su vez, es posible obtener una muestra profunda del tejido endometrial (Terttu K, 2016). A partir de un estudio realizado con 2123 hisopados endometriales se reportó un $11 \%$ de cultivos con aislamiento positivo, de los cuales, la bacteria más común fue Streptococcus $\beta$-hemolítico, con un $34 \%$, seguida por E. coli, con un $17 \%$ y por Pseudomonas spp., con un 12\% (Riddle WT, 2007).

El lavado de bajo volumen se ha indicado por su mayor precisión, en comparación con el hisopado uterino, en tanto todo el líquido recubre el área superficial del endometrio, por lo que el procedimiento garantiza un área mayor de muestreo. Éste se realiza, generalmente, con un volumen de 20 a $250 \mathrm{ml}$ (Ball BA, 1988), siendo normal la recuperación de volúmenes, a partir de 30 a $60 \mathrm{ml}$ (LeBlanc MM, 2007). Estudios realizados en los cuales la técnica utilizada en el 
diagnóstico fue el lavado de bajo volumen, indicaron un asilamiento bacteriano positivo en el $70 \%$ de las muestras; en estas el $42 \%$ correspondió a E. coli y el $38 \%$ a Streptococcus $\beta$ hemolítico (LeBlanc M.M., 2007). Teniendo en cuenta lo anterior, el presente estudio tuvo por objetivo realizar una comparativa en el aislamiento bacteriano uterino, entre las técnicas de hisopado y de lavado de bajo volumen, en yeguas criollas colombianas.

\section{METODOLOGÍA}

\section{Aspectos éticos}

El estudio se realizó bajo la normativa de la Ley 1774 del 6 de enero del 2016; de acuerdo con ello, se aseguró que ninguno de los animales objeto del presente estudio registrara algún signo de incomodidad o malestar. El proceso de levantamiento de la información fue desarrollado en el marco de ejercicios de diagnóstico clínico, los cuales fueron realizados por el profesional tratante de los especímenes; por lo anterior, no fue solicitado para dicho proceso un aval por parte de un comité de ética.

\section{Especímenes objeto de estudio}

Para el desarrollo del estudio se incluyeron 15 yeguas criollas colombianas, con edades entre los 4 a los 12 años, con una condición corporal de entre 2,5 a 4, según la escala propuesta por Webb y Weaver en 1979; dichos autores proponen un puntaje de 1 (muy pobre) a 6 (muy gordo), encontrándose los puntajes entre 3 o 4, clasificados como buenos, con registro de repetición de celo e historial de subfertilidad y signos clínicos y reproductivos de endometritis bacteriana.

\section{Clasificación de la endometritis}

Durante la realización de la presente investigación se identificó a la endometritis clínica, como el proceso de infección uterina con signos evidentes de descarga vaginal y acúmulo de exudado en la comisura ventral de la vulva. La endometritis subclínica se consideró como el proceso de infección uterina sin signos visibles, pero con historial de infertilidad y con posterior diagnóstico, a través de las pruebas utilizadas.

\section{Cultivo bacteriológico}

Se utilizaron dos técnicas para la toma de muestras endometriales. Una, mediante un hisopo double-guarded estéril especial para la especie equina, protegido con una funda para evitar su contaminación en su paso por la vagina y el cérvix; las muestras se obtuvieron de la porción dorsal y ventral de las paredes del cuerpo y base de los cuernos del útero y fueron enviadas en refrigeración, por medio de transporte Stuart, con el fin de asegurar su adecuada conservación. La otra técnica se realizó por medio del lavado de bajo volumen, utilizando una sonda Foley estéril No. 24, para la infusión de $150 \mathrm{ml}$ de solución salina fisiológica, de los cuales, se recuperaron $50 \mathrm{ml}$ en tubos eppendorf, los que fueron rotulados y almacenados bajo refrigeración, para su posterior transporte. Las dos técnicas fueron realizadas en el mismo momento; primero se tomó la muestra con el hisopo y luego se procedió con el lavado de bajo 
volumen. Además, se aseguró que las muestras llegaran en un período menor a 6 horas al laboratorio de destino, para su posterior análisis.

\section{Procesamiento de las muestras}

Las muestras obtenidas para el análisis y el aislamiento bacteriano fueron enviadas a un laboratorio, para su procesamiento y cultivo. Estas, a su vez fueron sembradas en agar MacConkey a una temperatura de $37^{\circ} \mathrm{C}$ y con un tiempo de espera de crecimiento bacteriano de 72 horas; para la clasificación de las colonias se utilizaron rutas bioquímicas (TSI, LISINA, UREA, CITRATO y SIM). La técnica de antibiograma se realizó bajo los estándares internacionales CLSI (Instituto de Estándares Clínicos y de Laboratorio).

\section{Análisis estadístico}

Se realizaron medidas de estadística descriptiva, en las cuales se determinó la frecuencia de la presentación de bacterias a nivel uterino en yeguas, utilizando una base de datos categorizada en hoja de cálculo Excel.

\section{RESULTADOS Y DISCUSIÓN}

La mayoría de los problemas de subfertilidad en las yeguas se asocian con la incapacidad de eliminar desechos inflamatorios, microflora contaminante y detritos postmonta (Leblanc MM, 2003). Desde los estudios realizados por Frontoso et al. (2008) se ha mencionado una alta incidencia de infecciones bacterianas a nivel reproductivo en yeguas; a partir de estos datos se obtuvieron resultados de entre el 50\% y el 70\%. Autores como Benko et al. (2015) indican una tendencia al aumento de las infecciones uterinas en yeguas, y apoyan su teoría al indicar incidencias en un 70\%, en los cultivos obtenidos mediante hisopos uterinos (Benko T, 2015). Lo antes mencionado concuerda con los resultados encontrados en el actual estudio, en el cual se identificó el aislamiento bacteriano positivo, para cada una de las 15 muestras analizadas y obtenidas mediante hisopado y lavado de bajo volumen.

En todo caso, resulta importante realizar un diagnóstico preciso, relacionando los signos clínicos y el diagnóstico microbiológico, con el fin de predecir el rendimiento reproductivo de las yeguas (Overbeck W, 2011). Diel de Amorim, et al. (2015) enfatizan en la importancia de realizar diagnósticos de endometritis en yeguas, basándose en múltiples parámetros clínicos, tales como la citología y la biopsia; ello, en tanto consideran que, de limitarse al cultivo, se puede llegar a obtener falsos negativos, debido a mecanismos de resistencia bacteriana, como la producción biolfim y la profundidad en el tejido en el que se encuentren las colonias bacterianas (Diel de Amorim M, 2015).

Ahora bien, de las 15 muestras evaluadas para el cultivo, mediante la técnica de hisopado, se encontró que $E$. coli se aisló en un 33,33\% (5/15); la asociación E. coli + Streptococcus spp. en un $13,33 \%(2 / 15)$ y E.coli + Staphylococcus spp. en un 13,33\% (2/15). Otras bacterias fueron aisladas de forma individual en menor proporción, como es el caso de Pseudomonas spp., en un $6,67 \%(1 / 15)$ (Tabla 1). 
Tabla 1.

Relación de aislamientos bacterianos mediante la técnica de hisopado uterino.

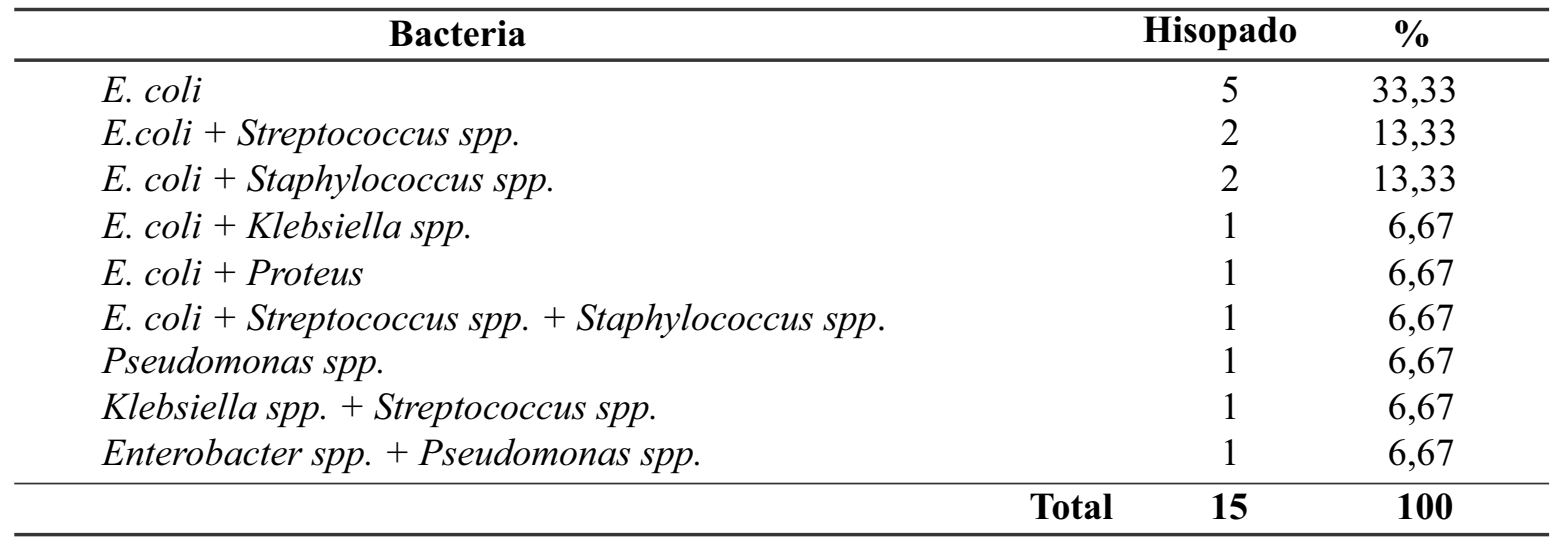

En otro orden de cosas, de las muestras conseguidas se obtuvo que E. coli y Pseudomonas spp., con el 13,33\% cada una, fueron las bacterias con mayor aislamiento de manera individual; así mismo, se encontró en mayor asociación a E. coli + Pseudomonas spp. + Klebsiella spp., y E. coli + Enterobacter spp., cada asociación con el 13,33\% (Tabla 2).

Tabla 2.

Relación de aislamientos bacterianos mediante la técnica de lavado de bajo volumen.

\begin{tabular}{|c|c|c|}
\hline Bacteria & Lavado & $\%$ \\
\hline E. coli & 2 & 13,33 \\
\hline E. coli + Enterobacter spp. & 2 & 13,33 \\
\hline E. coli + Pseudomonas spp. + Klebsiella spp. & 2 & 13,33 \\
\hline Pseudomonas spp. & 2 & 13,33 \\
\hline E. coli + Klebsiella spp. & 1 & 6,67 \\
\hline E. coli + Pseudomonas spp. & 1 & 6,67 \\
\hline E. coli + Pseudomonas spp. + Proteus & 1 & 6,67 \\
\hline E. coli + Pseudomonas spp. + Streptococcus spp. & 1 & 6,67 \\
\hline E. coli + Pseudomonas spp. + Klebsiella spp. + Staphylococcus spp. & 1 & 6,67 \\
\hline Streptococcus spp. + Klebsiella spp. + Pseudomonas spp. & 1 & 6,67 \\
\hline Streptococcus spp. + Pseudomonas spp. & 1 & 6,67 \\
\hline Total & 15 & 100 \\
\hline
\end{tabular}

A su vez, Albhin et al. (2003) registraron un aislamiento del 67\% para Echerichia coli, mientras que para Streptococcus $\beta$-hemolítico, el registrado fue del $20 \%$ en muestras para cultivo, tomadas mediante hisopado. Estos resultados son similares a los descritos por Morales y Castro (2018), quienes obtuvieron muestras uterinas para cultivo mediante la técnica de hisopo, reportando el aislamiento de $E$. coli en un $80 \%$ de los casos de endometritis. Lo anterior, concuerda con los resultados obtenidos en el presente estudio, en el cual se aisló con 
mayor frecuencia E.coli, y con menor frecuencia a las bacterias como Streptococcus spp., Staphylococcus spp., y Pseudomonas spp.

Con relación a la confirmación de casos de endometritis bacterianas, por cultivos, Beehan et al. (2015) afirman que son varias las causas por las cuales, algunas muestras podrían presentan falsos negativos; entre estas, mencionan por ejemplo que bacterias como E. coli tienen la facilidad de adherirse firmemente al epitelio, lo cual las hace difíciles de cultivar, si se utilizan técnicas como el lavado de bajo volumen, y la capacidad de crear biopeliculas protectoras, lo que finalmente dificulta su aislamiento.

Por otra parte, para el análisis comparativo se encontró un mayor aislamiento de $E$. coli mediante la técnica de hisopado, con un 33,33\% (5/15), respecto del lavado de bajo volumen, que presentó un porcentaje del 13,33\% (2/15). A su vez, E. coli, asociada a infecciones mixtas, se aisló con mayor frecuencia en el lavado de bajo volumen, con un 60\% (9/15), mientras que, mediante el hisopado, se identificó con un 46,67\% (7/15). Streptococcus spp. fue aislado en infecciones mixtas encontrando una mayor frecuencia, mediante el lavado de bajo volumen, con un 13,33\% (2/15), frente a su identificación por hisopado, en un 6,67\% (1/15) (Tabla 3$)$.

Tabla 3.

Comparativa de aislamientos bacterianos mediante las técnicas intrauterinas de hisopado y lavado de bajo volumen.

\begin{tabular}{lcccr}
\hline \multicolumn{1}{c}{ Bacteria } & Hisopado & $\mathbf{\%}$ & Lavado & \% \\
\hline E. coli & 5 & 33,33 & 2 & 13,33 \\
E. coli + infección mixta & 7 & 46,67 & 9 & 60 \\
Streptococcus spp. & 0 & 0,00 & 0 & 0,00 \\
Streptococcus spp. + infección mixta & 1 & 6,67 & 2 & 13,33 \\
Pseudomonas spp. & 1 & 6,67 & 2 & 13,33 \\
Pseudomonas spp. + infección mixta & 1 & 6,67 & 0 & 0,00 \\
\hline \multicolumn{1}{r}{ Total } & $\mathbf{1 5}$ & $\mathbf{1 0 0}$ & $\mathbf{1 5}$ & $\mathbf{1 0 0}$ \\
\hline
\end{tabular}

Por contraste, los resultados obtenidos en el estudio difieren de los reportados por Christoffersen et al. (2015), quienes, a pesar en coincidir en estos al reportar a $E$. coli como la bacteria con mayor frecuencia respecto de otras, indicaron su aislamiento en un $30 \%$, respecto de las muestras obtenidas con lavado de bajo volumen, y en un $12 \%$, respecto de las obtenidas mediante hisopado. La diferencia entre los porcentajes de reporte, a partir de la técnica utilizada para la obtención de la muestra, podría encontrarse relacionada con la capacidad del hiposo para obtener una muestra de mayor profundidad del epitelio endometrial.

Finalmente, vale indicar que Petersen et al. (2015) afirman que muchos resultados de cultivos presentan bajos valores de aislamiento, debido al alto número de yeguas con infecciones latentes por Streptococcus equi zooepidemicus, patógeno que reside profundamente en el endometrio, facilitando infecciones persistentes subclínicas. Lo indicado por los autores 
podría relacionarse con los resultados del presente estudio, en el que no se registró el aislamiento individualizado de Streptococcus equi zooepidemicus, bajo ninguna de las técnicas utilizadas, pero sí en combinación con otras bacterias, las cuales podrían asociarse a infecciones agudas, en las que la bacteria se encuentra a nivel superficial en el epitelio endometrial. Así las cosas, según el criterio de los autores, resulta necesario el desarrollo de estudios que involucren un mayor número de muestras y de la confirmación de los hallazgos, mediante la realización biopsias.

\section{CONCLUSIONES}

Las yeguas criollas colombianas registran una alta frecuencia en la presentación de endometritis bacteriana; el agente aislado más comúnmente encontrado, de manera individual y en infecciones mixtas, es $E$. coli. La técnica de hisopado demostró mayor eficacia en el aislamiento de $E$. coli, con respecto al lavado de bajo volumen; a su vez, las dos técnicas no resultaron efectivas para la identificación de Streptococcus equi zooepidemicus.

\section{REFERENCIAS BIBLIOGRÁFICAS}

Albihn, A.B., Baverud, V., Magnusson, U. (2003). Uterine microbiology and antimicrobial susceptibility in isolated bacteria from mares with fertility problems. Acta Veterinaria Scandinavica, 44, 121-129. doi: 10.1186/1751-0147-44-12

Ball, B. A., Shin, S. J., Patten, V. H, Lein, D. H., Woods, G. L. (1988). Use of a low-volume uterine flush for microbiologic and cytologic examination of the mare's endometrium. Theriogenology, 29, 1269-83. doi.org/10.1016/0093-691X(88)90007-6

Beehan, D. P., Wolfsdort, K., Elam, J., Krekeler, N., Paccamonti, D., Lyle, S. (2015). The evaluation of biofilm-forming potential of Escherichia coli collected from the equine female reproductive tract. J Equine Vet Sci (35), 935-939. doi.org/10.1016/j.jevs.2015.08.018

Benko, T., Boldizar, M., Novotny, F., Hura, V., Valocky, I., Dudrikova, K., Karamanova, M., Petrovic, V. (2015). Incidence of bacterial pathogens in equine uterine swabs, their antibiotic resistance patterns, and selected reproductive indices in English thoroughbred mares during the foal heat cycle. Veterinarni Medicina, 11, 613-620. doi:10.17221/8529-VETMED

Causey, R. C., Miletello T, O’Donell L, Lyle S, Paccamonti D, Anderson K, Eilts B, Morse S, LeBlanc MM. (2008). Pathologic Effects of Clinical Uterine Inflammation on the Equine Endometrial Mucosa. THERIOGENOLOGY,(54), 276-277.

Christoffersen, M., Brandis, L., Samuelsson, J., Bojesen, A.M., Troedsson, M.H., Petersen, M.R. (2015). Diagnostic double-guarded low-volume uterine lavage in mares. Theriogenology, 83, 222-7. doi.org/10.1016/j.theriogenology.2014.09.008

Diel de Amorim, M., Gartely, C., Foster, R., Hill, A., Scholtz. E., Hayes. A., Chenier, T.. (2015). Comparison of clinical signs, endometrial culture, endometrial cytology, uterine low volume lavage, and uterine biopsy, and combinations in the diagnosis of Equine Endometritis. Journal of 
Equine Veterinary Science, 44, 54-61. doi:10.1016/j.jevs.2015.10.012

Ferris, R. A. (2016). Diagnostic tools for infectious endometritis. Vet Clin North Am Equine Pract, 32(3), 481- 498. doi:10.1016/j.cveq.2016.08.001

Frontoso, R., De Carlo, Pasolini, M. P., Meulen, K., Pagnini., U, Lovane, G., Martino., L. (2008). Retrospective study of bacterial isolates and their antimicrobial susceptibilities in equine uteri during fertility problems. Research in Veterinary Science, 8, 1-6. doi:10.1016/j.rvrsc.2007.02.008

LeBlanc, M. M. y Causey R., C. (2009). Clinical and subclinical endometritis in the mare: Both Threats to Fertility. Reprod Domest Anim, 44(3), 10 - 22 http://www.ncbi.nlm.nih.gov/pubmed/19660076

LeBlanc M. M., Magsig J., Stromberg, A. (2007). Use of a low-volume uterine flush for diagnosing endometritis in chronically infertile mares. Theriogenology, 68(3), 403-12. doi: 10.1016/j.theriogenology.2007.04.038

Leblanc, M. M. (2003). Persistent mating induced endometritis in the mares: pathogenesis, diagnosis and treatment. Recent Advances in Equine Reproduction. En B. B., A. (Ed.), Recent Advances in Equine Reproduction. International Veterinary Information Service.

Morales, P. C., Castro, R., A. (2018). Estimación de la integridad uterina en yeguas Pura Raza Chilena y su asociación con edad y número de partos. Rev Inv Vet Perú, 29(2), 565-574. doi:10.15381/rivep.v29i2.14489

Overbeck, W., Witte, T., S., Heuwieser, W. (2011). Comparison of three diagnostic methods to identify subclinical endometritis in mares. Theriogenology, 75, 1311-1318. doi:10.1016/j.theriogenology.2010.12.002

Petersen, M. R., Nielsen, J. M., Lehn-Jensen, H., Bojensen, A. M. (2009). Streptococcus equi subsp. zooepidemicus resides deep in the chronically infected endometrium of mares. Clin Theriogenology, 1, 393-409.

Petersen, M. R., Skive, B., Christoffesen, M., Lu K., Nielsen, J. M,, Troedsson, M.H., Bojesen, A. M. (2015). Activation of persistent Streptococcus equi subspecies zooepidemicus in mares with subclinical endometritis. Vet Microbiol, 179, 119-25. doi:10.1016/j.vetmic.2015.06.006

Riddle, W.T., Leblanc, M. M., Stromberg. A. (2007). Relationships between uterine culture, cytology and pregnancy rates in a Thoroughbred practice. Theriogenology, 68(3), 395-402. doi: 10.1016/j.theriogenology.2007.05.050

Scoggin, Ch. (2016). Endometritis, nontraditional therapies. Vet Clin North Am Equine Pract, 32(3): 499-511. doi: 10.1016/j.cveq.2016.08.002

Siemieniuch, M. J., Kozdrowski R., Mioduchowska S., Nowak, R. (2019). Evidence for Increased Content of PGF2a, PGE2, and 6-keto-PGF1a in endometrial tissue cultures from heavy draft mares in anestrus with endometritis. Journal of Equine Veterinary Science, 77, 107 - 113. doi: 10.1016/j.jevs.2019.02.014Terttu, K. (2016). Evaluation of diagnostic methods in equine endometritis. Reprod Biol, 16, 189-196. doi:10.1016/j.repbio.2016.06.002. 